\title{
SEARCHING AND TRACKING OF NEIGHBORING BASE STATIONS
}

\author{
S. Jacqulin Veda Jancy ${ }^{1}$, B. Jesvin Veancy ${ }^{2}$, P.Yogesh ${ }^{3}$ \\ ${ }^{1}$ M.E Communication Systems, Easwari Engineering College, India \\ ${ }^{2}$ Assistant professor (Sl Gr), Easwari Engineering College, India \\ ${ }^{3}$ Dept of Information Science and Technology, Anna University, Chennai, India
}

\begin{abstract}
Long term evolution (LTE) is considered to be a key technology for the next generation of cellular telecommunications. Searching and tracking of neighboring cells is important for cellular network management such as handover and base station cooperation. The cell search procedure begins when the mobile unit is powered on and it starts communicating with the strongest base station. The main objective is to find the neighbor cells in the LTE system. Simulations are done using LTE simulator to find the probability of detecting the number of neighbor cells under various channel conditions and at different UE locations.
\end{abstract}

Keywords: Long Term Evolution (LTE), Cell search, synchronization, PSS, SSS.

\section{INTRODUCTION}

The Long Term Evolution (LTE) is an upcoming mobile communication standard that is specified by the 3rd Generation Partnership Project (3GPP). LTE is a wireless broadband technology designed to support roaming Internet access via cell phones and handhelds. The goal of LTE is to provide low-latency, high-data-rate and packet-optimized radio-access technology. LTE system supports peak data rates of $326 \mathrm{Mb} / \mathrm{s}$ with 4 X $4 \mathrm{MIMO}$ within $20 \mathrm{MHz}$ bandwidth. LTE system supports flexible bandwidths. It supports OFDMA and SC-FDMA access schemes. In addition to FDD (Frequency Division Duplexing) and TDD (Time Division Duplexing), half duplex FDD is also allowed to support low cost UE. OFDMA allows data to be directed to or from multiple users on a subcarrier - by - subcarrier basis for a specified number of symbol periods. The SC-FDMA has much lower PAPR(Peak to Average Power Ratio) when compared to OFDMA which makes it a great choice for uplink transmission in LTE. In mobile cellular systems, initial cell search is an important task for a user equipment (UE) to get synchronized to an eNode B (eNB) when the UE starts up. Reliable searching and tracking of neighboring cells around the user equipment (UE) in addition to the serving cell provides rich information for cellular network management and applications, such as handover, base station cooperation, and location inference. For example, reliable cell detection (i.e., with a high detection probability) leads to efficient handover of the UE to the best neighboring cell. The number of detected cells is also an important metric for many applications: (i) the performance of UE's location inference highly depends on the number of detected cells [4] (ii) in heterogeneous network deployment more detected cells will give the base station a larger flexibility to offload the traffic and optimize the network throughput via inter-cell interference coordination (ICIC).Cell identity (ID) information in LTE systems is carried by the signals in both the primary and secondary synchronization channels called primary and secondary synchronization signals (PSS and SSS) respectively. While the PSS is mainly dedicated for synchronization and the SSS provides complete information about the cell ID.

\section{CELL SEARCH}

In the LTE system the mobile unit has to search for the available radio cells and lock to one of them to continue communication while moving between the cells. Multiple mobile unit users simultaneously try to access the same set of radio cells and also the UE mobile unit begins it search blindly without any knowledge of the bandwidth it has allocated. Hence the initial cell search procedure must also be implemented in timing and frequency synchronization[1].

UE will tune its radio turn by turning to different frequency channels depending upon which bands it is supporting. Assuming that it is currently tuned to a specific band / channel UE first finds the primary synchronization signal (PSS) which is located in the last OFDM symbol of first time slot of the first sub frame (sub frame 0) of radio frame. This enables UE to be synchronized on sub frame level. The PSS is repeated in sub frame 5 which means UE is synchronized on $5 \mathrm{~ms}$ basis since each sub frame is $1 \mathrm{~ms}$.UE is also able to obtain physical layer identity ( 0 to 2 ) from PSS. In the next step UE finds the secondary synchronization signal (SSS). SSS symbols are also located in the same sub frame of PSS but in the symbol before PSS. From SSS UE is able to obtain physical layer cell identity group number (0 to 167 ).

Using physical layer identity and cell identity group number, UE knows the cell ID for the cell . LTE systems consist of 504 unique physical layer cell identities. To accommodate and 
manage this large amount, the cell identities are divided into 168 unique cell layer identity groups. Each group further consists of three physical layer identities. This is usually represented as: $\mathrm{N}_{(1)} \mathrm{ID}=0 \ldots 167$ and $\mathrm{N}_{(2)} \mathrm{ID}=0,1,2$.

$$
\text { Cell ID is calculated as } 3 \times \mathrm{N}_{(1)} \text { CellID }+\mathrm{N}_{(2)} \text { CellID }
$$

In LTE system there are 504 Physical Cell Identities. LTE can address 504 cell identity with the PSS and SSS . In the LTE air interface, Physical Layer Cell Identity is used for cell identification and channel synchronization. The $\mathrm{N}_{(1)}$ CellID and $\mathrm{N}_{(2)}$ CellID values are also used to seed the Secondary Synchronization Signal and Primary Synchronization Signal. The cell identity is a number from 0 to 503 and it distinguishes a cell from its immediate neighbors.

\section{SYNCHRONIZATION SIGNALS DETECTION}

The hierarchical cell search procedure is performed in two steps using two signals detection:

1. Primary Synchronization Signal and

2. Secondary Synchronization Signal

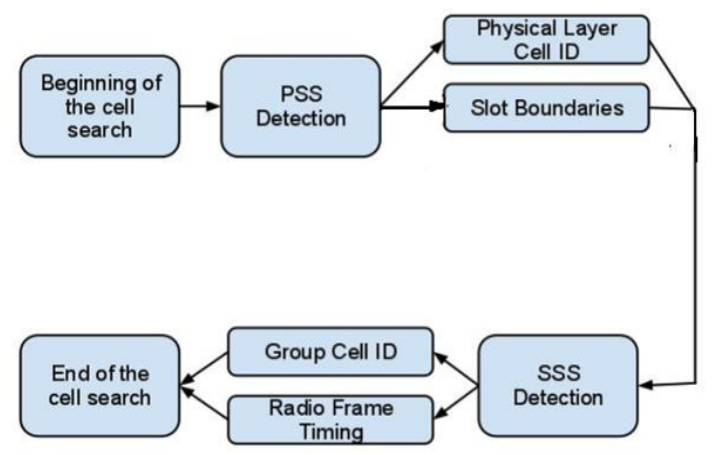

Fig 1 Cell Search

The synchronization signals have 72 subcarriers reserved for them, but they use only 62 of the 72 subcarriers. The Primary Synchronization signal first determines one of three cell identities $(0,1,2)$ and it is also represented by $\mathrm{N}_{(2)} \mathrm{ID}$. The secondary synchronization signal is used to determine a cell ID between 0 and 167 represented by $\mathrm{N}_{(1)} \mathrm{ID}$.

\subsection{PSS Detection}

The main function of PSS is to detect the boundary of a frame where non-coherent detection method has to be used at the receiver since there is no known reference information initially. Matched filter is a basic non - coherent detection method that can be used to detect PSS efficiently. The sequence is mapped to the subcarriers around DC and transformed into time domain by 64bit IDFT[4]. To detect this signal at the receiver, the correlation with the domain signal of the $\mathrm{ZC}$ sequence is calculated. If the maximum value of correlation is greater than a preset threshold, then corresponding OFDM symbol timing is noted and the symbol is assumed to contain the PSS corresponding to the reference sequence. The PSS value is provided by index of the correlation maximizing reference sequence. The UE knows which PSS sequence is being received and it has a known reference of the signal, the UE can now estimate the channel using its known reference of the original signal.

At the end of this step the UE knows:

1. Slot boundary.

2. Cell ID index (PSS value)

In FDD system the PSS is transmitted in sub frame 0 or sub frame 4. So with the detection of PSS, the UE knows it is synchronized with either sub frame 0 or sub frame 5 . Determination of whether it is sub frame 0 or sub frame 5 will enable frame timing synchronization which will be performed with the detection of SSS.

\subsection{SSS Detection}

The SSS is transmitted by the same subcarriers as those of PSS in the immediately preceding symbol. Therefore we can assume that these channel parameters remain the same for the SSS subcarriers as well. The SSS is a binary sequence of +1 's \& -1's formed after the interleaving of two sequences. Furthermore these two sequences are scrambled with two binary sequences which depend on $\mathrm{N}_{2}$ (ID) . Therefore the detection process proceeds by first deriving the scrambling sequences, then finding the values and then finally computing the value by a simple table look-up procedure. The sequence $\mathrm{d}(0) \ldots . . . \mathrm{d}(61)$ used for the secondary synchronization signal is an interleaved concatenation of two length-31 binary sequences. The concatenated sequence is scrambled with a scrambling sequence given by the PSS. The combination of two length-31 sequences defining the secondary synchronization signal differs between sub frame 0 and sub frame 5 according to:

$$
\begin{aligned}
& \mathrm{d}(2 \mathrm{n})=\mathrm{s}_{0}{ }^{(\mathrm{m} 0)}(\mathrm{n}) \mathrm{c}_{0}(\mathrm{n}) \text { in slot0 } \\
& \mathrm{d}(2 \mathrm{n})=\mathrm{s}_{1}{ }^{(\mathrm{ml})}(\mathrm{n}) \mathrm{c}_{0}(\mathrm{n}) \text { in slot10 } \\
& \mathrm{d}(2 \mathrm{n}+1)=\mathrm{s}_{1}{ }^{(\mathrm{ml})}(\mathrm{n}) \mathrm{c}_{1}(\mathrm{n}) \mathrm{z}_{1}{ }^{(\mathrm{m} 0)}(\mathrm{n}) \text { in slot } 0 \\
& \mathrm{~d}(2 \mathrm{n}+1)=\mathrm{s}_{0}{ }^{(\mathrm{m} 0)}(\mathrm{n}) \mathrm{c}_{1}(\mathrm{n}) \mathrm{z}_{1}{ }^{(\mathrm{ml})}(\mathrm{n}) \text { in slot } 10
\end{aligned}
$$

The above equation clearly indicates that the SSS is different for sub frame 0 and sub frame 5. So, detection of SSS will enable UE to determine the frame timing as well. The detection of SSS is a coherent process. Since the UE has determined an estimate of the channel from the PSS, it now removes the effects of the channel before it detects the SSS. The SSS and PSS are closely located in time to enable the coherent detection. 
The block diagram in Figure 2 assists in descrambling the SSS signal. The following steps illustrate the detection of the SSS.

1. Channel effects are removed from the SSS and any frequency offset determined earlier is corrected.

2. The received signal is de-interleaved into the odd part and the even part, i.e. $d(2 n)$ and $d(2 n+1)$. Since $N_{(2)}$ ID is known from the PSS, the scrambling code $c_{0}(n)$ is already known to the UE and can be descrambled from the received signal. So the UE now correlates the descrambled signal with copies of $\mathrm{s}_{0}{ }^{(\mathrm{m} 0)}(\mathrm{n})$ to determine a match. Now, the UE has determined $\mathrm{m} 0$ or $\mathrm{m} 1$.

3. Now that the UE knows one value of $\mathrm{m} 0$ or $\mathrm{m} 1$, it can use it to descramble the sequence $\mathrm{z}$.

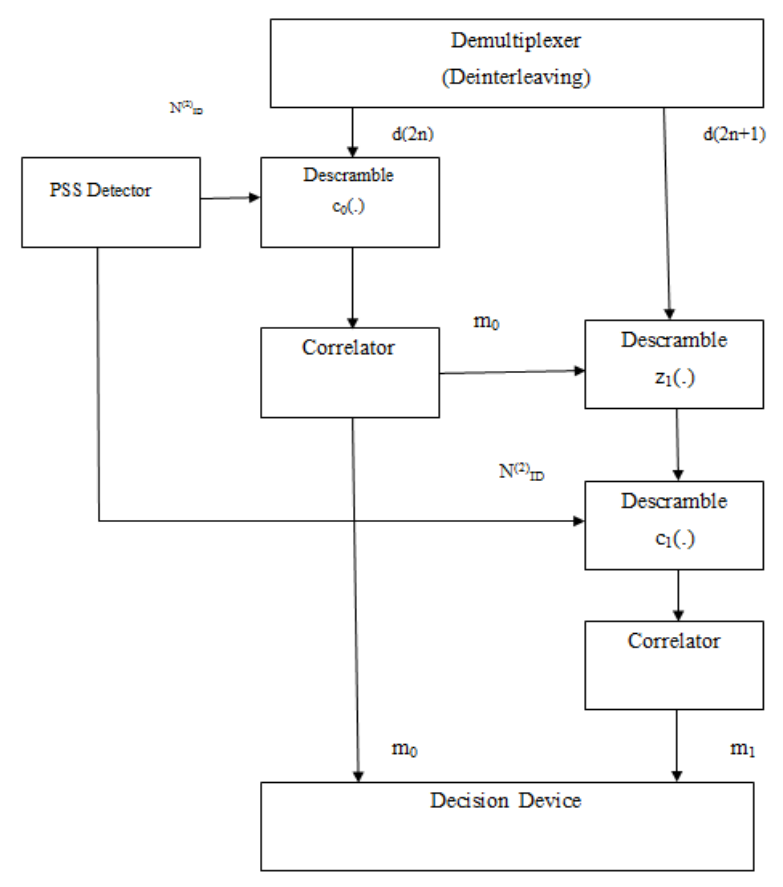

Fig 2 SSS Detection

4. The equations (1) to(4) is used to find the solution.

$$
\left.\mathrm{z}_{1}{ }_{\mathrm{o}}^{\mathrm{m}}\right)(\mathrm{n})=\mathrm{Z}\left(\left(\mathrm{n}+\left(\mathrm{m}_{0} \bmod 8\right)\right) \bmod 31\right)
$$

5. Say, the UE is in subframe zero, $\mathrm{c}_{0}$ was known to the UE, $\mathrm{m}_{0}$ was determined using $\mathrm{s}_{0}($.$) and using this \mathrm{m}_{0}, \mathrm{z}_{1}$ was descrambled. Similarly $\mathrm{m} 1$ is calculated. So, now the Cell Identity:

$$
\text { NcellID }=3 \mathrm{~N}(1) \mathrm{ID}+\mathrm{N}(2) \mathrm{ID}
$$

With this the UE is also able to determine the sub frame number, thus completing frame timing synchronization also. At the end of this step, the UE has determined the radio cell ID and Radio Frame value is determined. Thus the neighbor cell identity is determined.

\section{SIMULATION RESULTS}

The cell search in LTE System under different channel conditions is simulated in LTE system level simulator. We consider the deployment of 57 cells with wrap - around structure and inter - cell distance equal to $1.732 \mathrm{~km}$. The SNR is defined as the average received signal power at $1 \mathrm{~km}$ from the cell to the noise power ratio. The cell center, middle, and edge are referred to be the area within $0.4 \mathrm{~km}$, between $0.4 \mathrm{~km}$ to $0.7 \mathrm{~km}$, and between $0.7 \mathrm{~km}$ to $1 \mathrm{~km}$, to the cell site, respectively.

\section{Basic Parameters for LTE Simulation Environment}

$\begin{array}{ll}\text { Frequency } & 2.0 \mathrm{GHz} \\ \text { Receiver noise figure } & 9 \mathrm{~dB} \\ \text { System Bandwidth } & 5 \mathrm{MHz} \\ \text { Thermal noise density } & -174 \mathrm{dBm} / \mathrm{Hz} \\ \text { Inter eNodeB distance } & 1732 \mathrm{~m} \\ \text { Macroscopic pathloss } & 128.1+37.6 \log 10(\mathrm{R}) \\ \text { eNodeB TX Power } & 20 \mathrm{watts} \\ \text { UE speed } & 5 \mathrm{~km} / \mathrm{h} \\ \text { Transmission time interval } 100 \\ \text { Cyclic prefix }\end{array}$

\subsection{Number of Neighboring Cells in Flat Fading} Channel

In this the LTE network consists of $\mathrm{Nt}$ different cells with distinct cell IDs where $\mathrm{Nt}=57,100,200,504$.The probability of finding the number of neighbouring cells in flat fading channel is simulated.

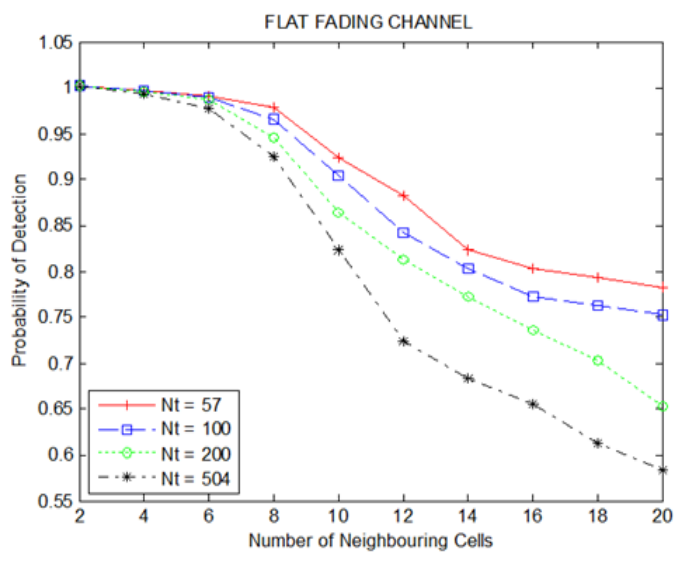

Fig 3 Probability of detection with respect to the number of neighboring cells in flat fading channel

It is shown that when the number of cells in a system is small $(\mathrm{Nt}=57)$ then the probability of neighbor cell detection is higher and when the systems cell number is large the probability of neighbor cell detection is reduced. 


\subsection{Number of Neighboring Cells in Multi Path} Fading Channel

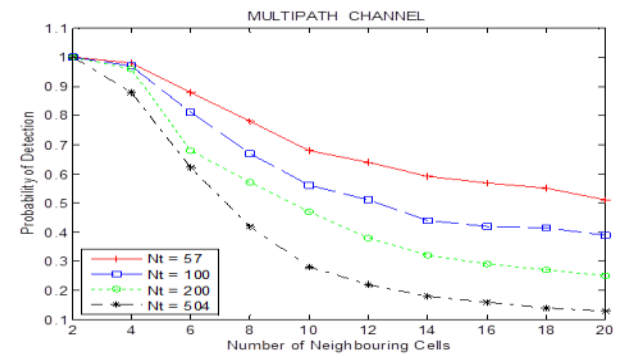

Fig 4 Probability of detection with respect to the number of neighboring cells in multi path fading channel

In this the LTE network consists of Nt different cells with distinct cell IDs where $\mathrm{Nt}=57,100,200,504$.The probability of finding the number of neighboring cells in multipath fading channel is simulated. It is shown that even when the number of cells in a system is small $(\mathrm{Nt}=57)$ the probability of neighbor cell detection is less when compared to flat fading channel. The probability of detection is less because of the reflection of radio waves by the obstacles present in the environment.

\subsection{Cell Detection with respect to SNR}

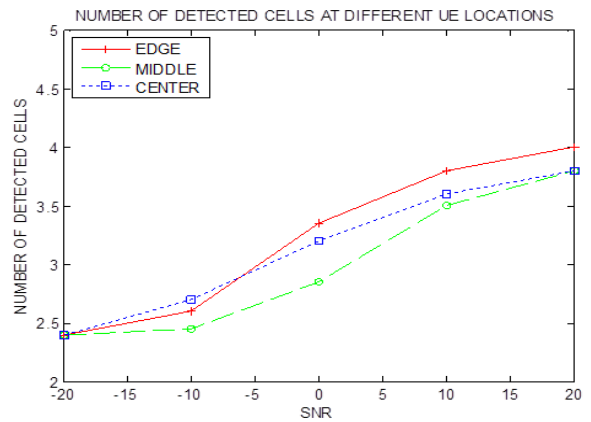

Fig 5 Number of detected cells at different UE locations (cell edge, middle and center) with respect to the SNR

In this the LTE network consists of 57 different cells with distinct cell IDs. The number of detected cells at different UE locations with respect to different SNR is simulated. The SNR is defined as the average received signal power at $1 \mathrm{~km}$ from the cell to the noise power ratio. The different UE locations are cell center, middle, and edge which are referred to be the area within $0.4 \mathrm{~km}$, between $0.4 \mathrm{~km}$ to $0.7 \mathrm{~km}$, and between $0.7 \mathrm{~km}$ to $1 \mathrm{~km}$, to the cell site, respectively. It is shown that the UE can detect most cells at the cell edge compared to at the cell center and at the cell middle in high SNR regimes, which agrees with intuition that the UE at cell edge has a better overall signal reception from its neighboring cells. However, in low SNR regimes, the better performance is obtained at cell center than at cell middle. This is because the UE at cell center can better estimate the channel to its serving cell and hence cancel its signal for other cell detection, and accurate detection and channel estimation for the first cell is especially important for other cells in low SNR regimes.

\subsection{Cell Detection with respect to the Number of Candidate Cells}

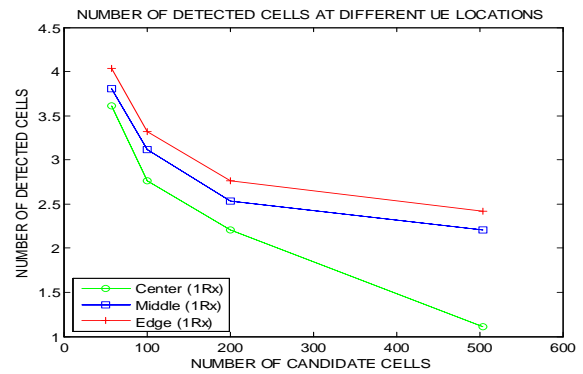

Fig 6 Number of detected cells at different ue locations (cell edge, middle and center) with respect to the number of candidate cells.

In this Number of detected cells at different UE locations (cell edge, middle, and center) with respect to the number of candidate cells is simulated. The UE is equipped with a single antenna and SNR $=20 \mathrm{~dB}$. When the UE is in edge both the number of detected cells and detection probability decreases with the increase in number of candidate cells The same is obtained for cell center and cell middle. This shows the effect of candidate cell set on the system. Thus the candidate cell number should be reduced in practical system to improve the performance.

\section{CONCLUSIONS AND FUTURE WORK}

This work offered an insight into synchronization and cell search in future LTE Mobile communication systems. Therefore two synchronization signals, the PSS and SSS are defined by 3 GPP. The PSS on the one hand allows precise estimation of the $\mathrm{N}_{2}$ (ID). This estimation is made possible through exploitation of the ideal cyclic auto - correlation properties of the used Zadoff-Chu sequences.In addition the base station allows it's identification by the SSS which allow the computation of the cell-ID. Through interleaving of multiple sequences, which have ID depending cyclic shifts the UE is able to estimate the IDs using correlation. Finally the neighbour cell search in LTE systems under various channel conditions and number of neighbour cells at different UE locations with respect to different SNR has been simulated. The above works has been generated by simulating the scenario in LTE System Level Simulator. This can be extended to perform interference reduction and handoff process when a UE moves form one cell to another in LTE system. 


\section{REFERENCES}

[1]. Cell search and cell selection in UMTS LTE by Rohde \& Schwarz.

[2]. Wikipedia. (2011). 3GPP Long Term Evolution

[3]. Synchronization and Cell Search Seminar LTE:: Derr Mobiillf funk derr Zukunfftt Fabian Schuh University Erlangen-Nürnberg Chair of Mobile Communications. January 2010.

[4]. 3GPP. (n.d.). 3GPP TR 25.913 - v7.3.0, Requirements for EUTRA and EUTRAN Available: http://www.3gpp.org/ftp/Specs/archive/25\%5Fseries/25

[5]. A. Roessler, Cell Search and Cell Selection in UMTS LTE. Munich, Germany: , 2009.

[6]. G. Li, and G. L. Stüber, Eds., Orthogonal Frequency Division Multiplexing for Wireless Communications. New York: Springer, 2006.

[7]. European Telecommunications Standards Institute, 3GPP TS 36.211 version 9.1.0 Release 9, Sophia Antipolis, France: ETSI, 2010. [15] H. G.Myung, J. Lim, and D. J. Goodman, "Single Carrier FDMA forUplink Wireless Transmission," IEEE Veh. Technol. Soc., vol. 1, no. 3, pp. 30-38, Sept. 2006. [8]. A Novel Low Complexity Cell Search Scheme for LTE Systems Pin-Kai Tseng, Sen-Hung Wang, Student Member, IEEE and Chih-Peng Li, Member, IEEE,. 